\title{
Efficacy of ceftazidime-avibactam in the treatment of infections due to Carbapenem-resistant Enterobacteriaceae
}

\author{
Basem M. Alraddadi ${ }^{1,2^{*}}$ (D), Mohammed Saeedi ${ }^{1}$, Mohammed Qutub ${ }^{3}$, Abeer Alshukairi ${ }^{1}$, Ashraf Hassanien ${ }^{4}$ and \\ Ghassan Wali ${ }^{1}$
}

\begin{abstract}
Background: Carbapenem-resistant Enterobacteriaceae (CRE) represent an important global threat. The aim of this study is to describe the clinical course and outcomes of patients with CRE infections treated with ceftazidimeavibactam (CAZ-AVI) compared to patients treated with other agents.

Methods: A retrospective cohort study of patients with established CRE infections from January 2017 until August 2018 was conducted. All patients who received CAZ-AVI and all cultures with carbapenem-resistant isolates were screened. We compared patients who received CAZ-AVI for CRE infections with patients who received other agents.

Results: A total of 38 consecutive patients with CRE infections were identified. Age and baseline comorbidities were similar between the two groups. The median time from admission to isolation of CRE culture was 22.5 days in the CAZ-AVI group and 17 days in the comparative group $(P=0.7)$. The incidence of CRE bacteremia was similar between the two groups: 7 patients (70\%) in the CAZ-AVI group and 15 patients (53.6\%) in the comparative group $(P=0.47)$. The most common type of CRE infections in both groups was hospital acquired pneumonia (HAP). Klebsiella pneumoniae was the predominant pathogen in both groups. A carbapenemase gene was detected in 35 (92\%) patients; the OXA-48 gene was the predominant gene identified in 28 (74\%) isolates. Eight out of ten patients in the CAZ-AVI group and fifteen out of twenty-eight in the comparative group achieved clinical remission ( $P=$ 0.14). After thirty days, all-cause mortality was observed in five patients in the CAZ-AVI group and 16 patients in the comparative group, accounting for 50 and $57 \%$ respectively.
\end{abstract}

Conclusions: In patients with established OXA-48-type CRE infection, CAZ-AVI is a reasonable alternative to standard therapy. These findings need to be confirmed in prospective studies.

Keywords: Ceftazidime-avibactam, Carbapenem-resistant Enterobacteriaceae, Saudi Arabia

\section{Background}

The emergence of carbapenem-resistant Enterobacteriaceae (CRE) represents a threat to global public health [1, 2]. Carbapenems are considered the last line of defence against Enterobacteriaceae. The burden of CRE infections is substantial, including longer lengths of stay, higher infection-related mortality and higher health care costs than those associated with carbapenem-susceptible

\footnotetext{
* Correspondence: balraddadi@kfshrc.edu.sa

${ }^{1}$ Department of Medicine, King Faisal Specialist Hospital and Research Center, Jeddah, Saudi Arabia

${ }^{2}$ Department of Medicine, University of Jeddah, Jeddah, Saudi Arabia Full list of author information is available at the end of the article
}

Enterobacteriaceae (CSE) [3-5]. Several studies have reported that carbapenem resistance is an independent risk factor for mortality, which is likely due to inappropriate initial antimicrobial therapy [6-8].

Treatment options for CRE infections are very limited. Polymyxins have been used for the treatment of CRE infections; however, there remain concerns regarding increasing resistance, limited efficacy and toxicity [9-12]. Novel $\beta$-lactam/ $\beta$-lactamase inhibitor combinations have emerged as new treatment options for CRE infections [12, 13]. However, these combinations are not active against all carbapenemases [13] . Ceftazidime-avibactam (CAZ-AVI) is a new $\beta$-lactam/ $\beta$-lactamase inhibitor combination with in vitro activity

C The Author(s). 2019 Open Access This article is distributed under the terms of the Creative Commons Attribution 4.0 International License (http://creativecommons.org/licenses/by/4.0/), which permits unrestricted use, distribution, and 
against Klebsiella pneumoniae carbapenemases (KPCs) and OXA-48 producing Enterobacteriaceae [14].

There is accumulating evidence of the utility of CAZ-AVI for the treatment of infections caused by resistant gram-negative bacilli, including CRE infections [15-19]. However, most of the published studies included patients with KPCs. A recent study documented the successful treatment of patients with OXA-48-type CRE infections with CAZ-AVI [20]. In Saudi Arabia, carbapenemases are highly prevalent in $K$ pneumoniae isolates. OXA- 48 is the predominant carbapenemase followed by New Delhi metallo- $\beta$ -lactamase (NDM) [21]. Our aim is to describe the clinical course and outcomes of patients with CRE infections treated with CAZ-AVI compared to those of patients treated with other agents.

\section{Methods}

We conducted a retrospective cohort study of patients with CRE infections from January 2017 to August 2018 at King Faisal Specialist Hospital and Research Center (KFSHRC), Jeddah, Saudi Arabia. All adult patients (> 18 years) who received CAZ-AVI for at least $24 \mathrm{~h}$ and all cultures with carbapenem-resistant isolates were screened. We included patients with clinically established CRE infections. Cases where CRE cultures likely represented colonization were excluded. CAZ-AVI was not available at our hospital until December 2017; we compared patients who received CAZ-AVI for the treatment of CRE infections between December 2017 and August 2018 with patients with CRE infections between January 2017 and November 2017 who received other CRE-specific therapies. Baseline characteristics were recorded, and clinical, microbiological and therapeutic data were collected. Clinical course and outcome data until death or hospital discharge were obtained. The primary outcome was complete remission, as evaluated by infectious disease specialists, defined as resolution of fever and eradication of bacteria in subsequent cultures. The secondary outcomes recorded were clinical cure without relapse or death within 30 days, 30-day mortality from starting CAZ-AVI, mortality due to CRE, length of stay in days and 30-day relapse rate with the same isolate.

Data extraction was performed by a trained physician and collected in a special case record form. Data included patients' demographics, Charlson comorbidity index, concomitant diseases, time from admission to first culture isolate of CRE culture in days, presence of bacteremia, type of infection, organism isolated, time from first CRE culture to starting CRE-specific therapy and CREspecific therapy used. Susceptibility testing was performed using the Vitek 2 system (bioMérieux, Marcy L'étoile, France) and N-291 card. Phenotypic conformation of CRE was performed using the Clinical Laboratory Standards Institute (CLSI) methodology, which includes the ertapenem, imipenem, and meropenem E-test and modified Hodge test (MHT). All confirmed isolates of CRE from the culture were then tested using the Xpert Carba- $\mathrm{R}$ Kit following the manufacture's recommendation for rapid detection and differentiation of the $b l a_{\mathrm{KPC}}, b l a_{\mathrm{NDM}}, b l a_{\mathrm{VIM}}, b l a_{\mathrm{OXA}-48}$ and $b l a_{\mathrm{IMP}}$ gene sequences linked to carbapenem resistance in gram-negative bacteria. The interpretation of the minimum inhibitory concentration (MIC) for carbapenems is based on CLSI guidelines; resistance to ertapenem was considered if MIC was $\geq 2 \mu \mathrm{g} / \mathrm{ml}$ and resistance to meropenem and imipenem was considered if MICs were $\geq 4 \mu \mathrm{g} / \mathrm{ml}$.

All data were analysed using IBM SPP version 25 . Continuous data were described using mean and standard deviation for the normally distributed data Medians and interquartile ranges were used for nonnormal data. Frequency and percentages were used to describe categorical data. Group comparison was performed using the by chi-square or Fisher's exact test for proportions. Student's $t$-test was used to compare continuous data. A $P$ value of $<0.05$ was considered significant.

\section{Results}

We identified 13 patients who received CAZ-AVI for the treatment of CRE infections between December 2017 and August 2018. Three patients were excluded from the analysis for being children (two patients) and being only treated with only CAZ-AVI for less than $24 \mathrm{~h}$ (one patient). The comparative group included 28 patients with CRE infections using the same criteria between January 2017 and November 2017 at KFSHRC-Jeddah. The median age was similar between the two groups: 59.5 years in the CAZ-AVI group and 61.5 years in the comparative group $(P=0.71)$. The median Charlson comorbidity index was 5.5 in the CAZ-AZI group compared to 5 in the comparative group $(P=0.86)$. The demographics and baseline characteristics for the remaining patients are included in Table 1 . Both treatment groups were similar, with no significant differences in terms of baseline data. OXA-48 was the predominant carbapenemase in patients who received CAZ-AVI $(8 / 10$, $80 \%$ ), one patient had NDM and in one patient, no carbapenemase gene was detected. In the comparative group, OXA-48 was the predominant carbapenemase as well (19/ 28, 68\%), 5 patients had NDM, one patient had both NDM and OXA-48, and no carbapenemase gene was detected in three patients. Carbapenem MIC distribution was similar between the two groups; details on mechanism of carbapenem resistance and antibiotic MICs for both groups are presented in Additional file 1: Table S1 and Additional file 2: Table S2. 
Table 1 Baseline characteristics of patients with CRE infections who received ceftazidime/avibactam compared with comparative group (received different CRE specific antibiotics)

\begin{tabular}{|c|c|c|c|}
\hline Characteristic & Ceftazidime/Avibactam group $n=10(\%)$ & Comparative group $n=28(\%)$ & $P$ value \\
\hline Male & $8(80)$ & $16(57.1)$ & 0.27 \\
\hline Age, median (IQR), y & $59.5(26-67)$ & $61.5(50-72)$ & 0.71 \\
\hline CCl, median (IQR) & $5.5(2-8.5)$ & $5(4-7.75)$ & 0.86 \\
\hline \multicolumn{4}{|l|}{ Baseline comorbidities } \\
\hline Diabetes mellitus & $4(40)$ & $15(53.6)$ & 0.71 \\
\hline Hypertension & $5(50)$ & $18(64.3)$ & 0.47 \\
\hline CVD & $4(40)$ & $9(32.1)$ & 0.71 \\
\hline Renal disease & $3(30)$ & $12(42.8)$ & 0.71 \\
\hline Malignancy & $5(50)$ & $7(25)$ & 0.24 \\
\hline Transplant & $5(50)$ & $5(17.9)$ & 0.09 \\
\hline HIV & 0 & $1(3.6)$ & $>0.99$ \\
\hline $\begin{array}{l}\text { Time from admission to first isolate of CRE culture } \\
\text { (days), median (IQR), days }\end{array}$ & $22.5(4.75-50.75)$ & $17(5.25-29.25)$ & 0.71 \\
\hline CRE Bacteremia & $7(70)$ & $15(53.6)$ & 0.47 \\
\hline \multicolumn{4}{|l|}{ Type of infection } \\
\hline CLABSI & $1(10)$ & $4(14.3)$ & $>0.99$ \\
\hline HAP & $5(50)$ & $14(50)$ & $>0.99$ \\
\hline CUTI & $3(30)$ & $8(28.6)$ & $>0.99$ \\
\hline $\mathrm{ClAl}$ & $3(30)$ & $5(17.8)$ & 0.41 \\
\hline SSTI & $2(20)$ & $3(10.7)$ & 0.59 \\
\hline \multicolumn{4}{|l|}{ Microbiology } \\
\hline Klebsiella pneumoniae & $7(70)$ & $23(82.1)$ & 0.41 \\
\hline Escherichia coli & $3(30)$ & $5(17.9)$ & \\
\hline $\begin{array}{l}\text { Time from first CRE culture to starting CRE specific } \\
\text { therapy, median (IQR), days }\end{array}$ & $3.5(1-8.75)$ & $0(0-1)$ & 0.05 \\
\hline
\end{tabular}

The median time (IQR) to the first isolation of CRE was 22.5 days (4.75-50.75) in the CAZ-AVI group compared to 17 days (5.25-29.25) in the comparative group. Hospital-acquired pneumonia (HAP) was the most common infection type in both groups, with $5(50 \%)$ patients in the CAZ-AVI group compared to 14 (50\%) in the comparative group (> 0.99). K. pneumoniae was the predominant pathogen in both groups. Details of baseline characteristics of both groups are included in Table 1. Groups remained similar after restricting analysis on patients with OXA-48 carbapenemase gene (Additional file 3: Table S3).

The comparative group received colistin (21, 75\%), carbapenem $(21,75 \%)$, tigecycline $(9,32.1 \%)$, aminoglycoside $(8,28.6 \%)$, quinolone $(4,14.3 \%)$, trimethoprim/ sulfamethoxazole $(1,3.6 \%)$ and aztreonam $(1,3.6 \%)$. Out of the 28 patients in the comparative group, 25 patients were administered antibiotic combinations. Details of the used combinations in the comparative group are presented in Additional file 4: Table S4.
Eight patients (80\%) in the CAZ-AVI group achieved clinical remission compared to 15 patients (53.6\%) in the comparative group $(P=0.14)$. The time to clearance of bacteremia was similar in both groups, with median (IQR) values of 4 days (3-5) and 5 days (3-7), respectively (Table 2). Thirty-day all-cause mortality and relapse with the same isolate were similar between the two groups $(5,50 \%)$ vs $(16,57.1 \%)(P=0.7)$ and $(2,20 \%)$ vs $(1,3.6 \%) \quad(P=0.1)$ respectively. Other secondary outcomes including relapse, attributable mortality and 30day mortality, were similar in both groups. There was no difference in outcome results after restricting analysis on patients with OXA-48 carbapenemase gene (Additional file 5: Table S5).

\section{Discussion}

Even though our small sample size likely precluded our ability to find statistically significant differences, our study demonstrated a clinically significant benefit of 
Table 2 Outcomes of patients with CRE infections who received ceftazidime-avibactam compared with comparative group (received different CRE specific antibiotics)

\begin{tabular}{llll}
\hline Outcome & Ceftazidime/Avibactam group $n=10(\%)$ & Comparative group $n=28$ (\%) & $P$ value \\
\hline Clinical remission & $8(80)$ & $15(53.6)$ & $11(39)$ \\
Clinical cure without relapse or & $4(40)$ & \\
death within 30 days & & $16(57.1)$ & $>0.14$ \\
$\quad 30$ days all-cause mortality & $5(50)$ & $11(39.3)$ & $40.5(22-79.5)$ \\
Attributable mortality to CRE & $2(20)$ & $1(3.6)$ & 0.7 \\
Length of stay, median (IQR), days & $69.5(47.5-96)$ & $5(3-7)$ & 0.27 \\
30-days relapse of the same isolate & $2(20)$ & & 0.1 \\
Time to clearance of bacteremia, & $4(3-5)$ & & 0.65 \\
median (IQR), days & &
\end{tabular}

CRE Carbapenem-resistant Enterobacteriaceae, IQR Interquartile range

CAZ-AVI for the treatment of CRE infections, including those caused by OXA- 48 producing organisms, compared to standard therapy. A majority of our patients had HAP infections. All-cause mortality in previously reported studies ranged from 24 to $39.5 \%$ compared to $50-57.1 \%$ in our cohort; we hypothesize that this difference is due to the high comorbidity index in our patients, reflecting the complex medical background and severe nature of these infections.

Many studies have investigated the role of various combination therapies for the treatment of CRE; two large retrospective studies showed that combination therapy (with two or more in vitro-active drugs, with meropenem in all patients) was associated with lower mortality rates than monotherapy (colisin, tigecycline, and gentamicin) in populations with high severity indices [22, 23].

CAZ-AVI has emerged as a promising therapy for CRE infections in several clinical studies, however, most of these studies included patients with KPCs [17, 24]. In a prospective multicenter cohort study to describe the clinical outcomes for patients with CRE infections compared, 38 patients treated with CAZ-AVI to 99 patients treated with colistin for KPC-producing CRE, it showed lower adjusted all-cause mortality in the CAZ-AVI group [24]. Sousa A. et al prospectively studied the effectiveness of CAZ-AVI as a rescue treatment for managing infections due to OXA-48-producing Enterobacteriaceae in 57 patients, $81 \%$ received CAZ-AVI as a monotherapy [20].

New treatment options for CRE infections have emerged in recent years, many of which exhibit activity against the KPC-producing gene but not OXA-48. Meropenem/vaborbactam is a novel $\beta$-lactamase inhibitor that exert activity against CRE by inhibiting class A carbapenemases such as KPCs but has no in vitro activity against class $B$ metallo- $\beta$-lactamases (NDM or VIM) or class D OXA 48 B-lactamases [25]. Plazomicin is a next- generation aminoglycoside that has been approved for the treatment of complicated urinary tract infections (cUTI). Studies have shown that plazomicin is more potent than other aminoglycosides against KPC-producing Enterobacteriaceae [12]. Eravasycline is a synthetic antibacterial agent of the tetracycline class that has been approved for the treatment of complicated intra-abdominal infections (cIAI) and has been shown to have twofold higher activity than tigecycline against CRE; however, there is limited clinical data for the efficacy of this drug against CRE infections [12]. Imipenem/cilastin and relebactam is a combination of imipenem and a novel $\beta$-lactamase inhibitor that exert activity against bacteria by inhibiting class A and $\mathrm{C}$ carbapenemases but has no in vitro activity against class $B$ metallo- $\beta$-lactamases or class D OXA-48 B-lactamasese. Cefiderocol is a novel cephalosporin with unique antibacterial activity against many CRE isolates and is active against carbapenemase hydrolysis [26].

The most prevalent carbapenemase-producing gene in Saudi Arabia is OXA-48, followed by NDM. In our cohort, a majority of the tested isolates carried the OXA48 gene [21]. Because of the different performances of these antimicrobial agents, we strongly stress on the importance of molecular testing to identify the gene responsible for causing the CRE infection and thus use the appropriate antibiotic.

The emergence of CAZ-AVI-resistant strains during treatment has already been reported and can be a contributing factor to increased mortality among patients with OXA-48 type CRE infections [16]. Shields et al described resistance to CAZ-AVI in 3 out of 10 patients with microbiological failure following treatment for 10 19 days. We believe that CAZ-AVI should be incorporated in standard antibiogram susceptibility testing.

Our study has several limitations. First, the retrospective nature of the analysis is a source of confounding by 
the indication type of bias. Additionally, due to the small sample size, our study was not sufficiently statistically powerful to detect a significant difference in efficacy or tolerability. Randomized controlled trials to address this vital issue are needed.

\section{Conclusions}

In summary, our study included a cohort of patients with invasive CRE infections, a majority of whom exhibited OXA-48 genotype, and showed that CAZ-AVI is a promising antibiotic for the treatment of these patients with limited therapeutic options. Despite the limitations and small size of the study, we were able to show that CAZ-AVI is effective and comparable to standard treatment for patients with established OXA-48-type CRE infections.

\section{Additional files}

Additional file 1: Table S1. Mechanism of carbapenem resistance and minimum inhibitory concentration for ceftazidime-avibactam group. (DOC $40 \mathrm{~kb}$ )

Additional file 2: Table S2. Mechanism of carbapenem resistance and minimum inhibitory concentration for the comparative group. (DOCX 15 $\mathrm{kb})$

Additional file 3: Table S3. Baseline characteristics of patients with OXA-48 CRE infections who received ceftazidime/avibactam compared with comparative group (received different CRE specific antibiotics) (DOC $51 \mathrm{~kb}$ )

Additional file 4: Table S4. Frequency of antibiotic combinations used for treatment of CRE infections in the comparative group. (DOCX $16 \mathrm{~kb}$ )

Additional file 5: Table S5. Outcomes of patients with OXA-48 CRE infections who received ceftazidime-avibactam compared with comparative group (received different CRE specific antibiotics). (DOC $41 \mathrm{~kb}$ )

\section{Abbreviations}

CAZ-AVI: Ceftazidime-Avibactam; CIAl: Complicated intra-abdominal infections; CLSI: Clinical Laboratory Standards Institute; CRE: Carbapenemresistant Enterobacteriaceae; CSE: Carbapenem-susceptible Enterobacteriaceae; CUTI: Complicated urinary tract infections; HAP: Hospital acquired pneumonia; IQR: Interquartile range; KPC: Klebsiella pneumoniae carbapenamase; MHT: Modified Hodge test; MIC: Minimum inhibitory concentration; NDM: New Delhi metallo- $\beta$-lactamase

\section{Acknowledgments}

Not applicable.

\section{Authors' contributions}

BA and MS conception and design, data acquisition, analytical plan, interpretation of data, drafting of the manuscript, critical revision of the manuscript for important intellectual content, approval of the final version to be published and agreement to be accountable e for all aspects of the work. $M Q, A A$ and GW data acquisition, critical revision of the manuscript for important intellectual content, approval of the final version to be published and agreement to be accountable e for all aspects of the work. AH critical revision of the manuscript for important intellectual content, approval of the final version to be published and agreement to be accountable e for all aspects of the work. All authors have read and approved the final manuscript.

\section{Funding}

Pfizer Biopharmaceutical contributed in language editing and articleprocessing charges. There is no role for the funding body in the design of the study and collection, analysis and interpretation of data.

\section{Availability of data and materials}

The datasets used and / or analyzed during the current study are available from the corresponding author on reasonable request.

Ethics approval and consent to participate

The study protocol was approved by the institutional review board of King Faisal Specialist Hospital and Research Center, Jeddah, Saudi Arabia. No administrative permission were required to access data.

\section{Consent for publication}

Not applicable.

\section{Competing interests}

$\mathrm{AH}$ works at Pfizer, anti infectives.

\section{Author details}

${ }^{1}$ Department of Medicine, King Faisal Specialist Hospital and Research Center, Jeddah, Saudi Arabia. ${ }^{2}$ Department of Medicine, University of Jeddah, Jeddah, Saudi Arabia. ${ }^{3}$ Department of Pathology and Laboratory Medicine, Section of Clinical Microbiology, King Faisal Specialist Hospital and Research Center, Jeddah, Saudi Arabia. ${ }^{4}$ Anti-infectives, Pfizer, Jeddah, Saudi Arabia.

Received: 27 May 2019 Accepted: 25 August 2019

Published online: 04 September 2019

\section{References}

1. Guidelines for the prevention and control of carbapenem-resistant Enterobacteriaceae, Acinetobacter baumannii and Pseudomonas aeruginosa in health care facilities. https://apps.who.int/iris/bitstream/handle/10665/25 9462/9789241550178-eng.pdf? sequence $=1$.

2. $\quad$ Lutgring JD. Carbapenem-resistant Enterobacteriaceae: an emerging bacterial threat. Semin Diagn Pathol. 2019:36(3):182-6.

3. Escandon-Vargas K, Reyes S, Gutierrez S, Villegas MV. The epidemiology of carbapenemases in Latin America and the Caribbean. Expert Rev Anti-Infect Ther. 2017;15(3):277-97.

4. Ben-David D, Kordevani R, Keller N, Tal I, Marzel A, Gal-Mor O, Maor Y, Rahav G. Outcome of carbapenem resistant Klebsiella pneumoniae bloodstream infections. Clinical microbiology and infection : the official publication of the European Society of Clinical Microbiology and Infectious Diseases. 2012; 18(1):54-60

5. Patel G, Huprikar S, Factor SH, Jenkins SG, Calfee DP. Outcomes of carbapenem-resistant Klebsiella pneumoniae infection and the impact of antimicrobial and adjunctive therapies. Infect Control Hosp Epidemiol. 2008; 29(12):1099-106

6. Villegas MV, Pallares CJ, Escandon-Vargas K, Hernandez-Gomez C, Correa A, Alvarez C, Rosso F, Matta L, Luna C, Zurita J, et al. Characterization and clinical impact of bloodstream infection caused by Carbapenemaseproducing Enterobacteriaceae in seven Latin American countries. PLoS One. 2016;11(4):e0154092

7. Kohler PP, Volling C, Green K, Uleryk EM, Shah PS, McGeer A. Carbapenem resistance, initial antibiotic therapy, and mortality in Klebsiella pneumoniae bacteremia: a systematic review and meta-analysis. Infect Control Hosp Epidemiol. 2017;38(11):1319-28.

8. Falagas ME, Tansarli GS, Karageorgopoulos DE, Vardakas KZ. Deaths attributable to carbapenem-resistant Enterobacteriaceae infections. Emerg Infect Dis. 2014;20(7):1170-5.

9. Yao X, Doi Y, Zeng L, Lv L, Liu JH. Carbapenem-resistant and colistinresistant Escherichia coli co-producing NDM-9 and MCR-1. Lancet Infect Dis 2016:16(3):288-9.

10. Liu YY, Wang Y, Walsh TR, Yi LX, Zhang R, Spencer J, Doi Y, Tian G, Dong B, Huang $X$, et al. Emergence of plasmid-mediated colistin resistance mechanism MCR-1 in animals and human beings in China: a microbiological and molecular biological study. Lancet Infect Dis. 2016;16(2): $161-8$.

11. Rojas LJ, Salim M, Cober E, Richter SS, Perez F, Salata RA, Kalayjian RC, Watkins RR, Marshall S, Rudin SD, et al. Colistin resistance in Carbapenem- 
resistant Klebsiella pneumoniae: laboratory detection and impact on mortality. Clinical infectious diseases : an official publication of the Infectious Diseases Society of America. 2017;64(6):711-8.

12. Sheu CC, Chang YT, Lin SY, Chen YH, Hsueh PR. Infections caused by Carbapenem-resistant Enterobacteriaceae: an update on therapeutic options. Front Microbiol. 2019;10:80.

13. Karaiskos I, Galani I, Souli M, Giamarellou H. Novel beta-lactam-betalactamase inhibitor combinations: expectations for the treatment of carbapenem-resistant gram-negative pathogens. Expert Opin Drug Metab Toxicol. 2019;15(2):133-49.

14. Shirley M. Ceftazidime-avibactam: a review in the treatment of serious gram-negative bacterial infections. Drugs. 2018;78(6):675-92.

15. Krapp F, Grant JL, Sutton SH, Ozer EA, Barr VO. Treating complicated carbapenem-resistant enterobacteriaceae infections with ceftazidime/ avibactam: a retrospective study with molecular strain characterisation. Int J Antimicrob Agents. 2017;49(6):770-3.

16. Shields RK, Potoski BA, Haidar G, Hao B, Doi Y, Chen L, Press EG, Kreiswirth $\mathrm{BN}$, Clancy CJ, Nguyen MH. Clinical outcomes, drug toxicity, and emergence of ceftazidime-avibactam resistance among patients treated for Carbapenem-resistant Enterobacteriaceae infections. Clinical infectious diseases : an official publication of the Infectious Diseases Society of America. 2016;63(12):1615-8.

17. Tumbarello M, Trecarichi EM, Corona A, De Rosa FG, Bassetti M, Mussini C, Menichetti F, Viscoli C, Campoli C, Venditti M, et al. Efficacy of ceftazidimeavibactam salvage therapy in patients with infections caused by Klebsiella pneumoniae Carbapenemase-producing K. pneumoniae. Clinical infectious diseases : an official publication of the Infectious Diseases Society of America. 2019;68(3):355-64.

18. Caston JJ, Lacort-Peralta I, Martin-Davila P, Loeches B, Tabares S, Temkin L, Torre-Cisneros J, Pano-Pardo JR. Clinical efficacy of ceftazidime/avibactam versus other active agents for the treatment of bacteremia due to carbapenemase-producing Enterobacteriaceae in hematologic patients. International journal of infectious diseases : IJID : official publication of the International Society for Infectious Diseases. 2017:59:118-23.

19. van Duin D, Bonomo RA. Ceftazidime/avibactam and Ceftolozane/ Tazobactam: second-generation beta-lactam/beta-lactamase inhibitor combinations. Clinical infectious diseases : an official publication of the Infectious Diseases Society of America. 2016;63(2):234-41.

20. Sousa A, Perez-Rodriguez MT, Soto A, Rodriguez L, Perez-Landeiro A Martinez-Lamas L, Nodar A, Crespo M. Effectiveness of ceftazidime/ avibactam as salvage therapy for treatment of infections due to OXA-48 carbapenemase-producing Enterobacteriaceae. J Antimicrob Chemother. 2018;73(11):3170-5.

21. Alotaibi F. Carbapenem-resistant Enterobacteriaceae: an update narrative review from Saudi Arabia. Journal of infection and public health. 2019

22. Tumbarello M, Trecarichi EM, De Rosa FG, Giannella M, Giacobbe DR, Bassetti M, Losito AR, Bartoletti M, Del Bono V, Corcione S, et al. Infections caused by KPC-producing Klebsiella pneumoniae: differences in therapy and mortality in a multicentre study. J Antimicrob Chemother. 2015:70(7):2133-43.

23. Gutierrez-Gutierrez B, Salamanca E, de Cueto M, Hsueh PR, Viale P, Pano-Pardo JR, Venditti M, Tumbarello M, Daikos G, Canton R, et al. Effect of appropriate combination therapy on mortality of patients with bloodstream infections due to carbapenemase-producing Enterobacteriaceae (INCREMENT): a retrospective cohort study. Lancet Infect Dis. 2017;17(7):726-34.

24. van Duin D, Lok JJ, Earley M, Cober E, Richter SS, Perez F, Salata RA, Kalayjian RC, Watkins RR, Doi Y, et al. Colistin versus ceftazidime-avibactam in the treatment of infections due to Carbapenem-resistant Enterobacteriaceae. Clinical infectious diseases : an official publication of the Infectious Diseases Society of America. 2018;66(2):163-71.

25. Castanheira M, Huband MD, Mendes RE, Flamm RK. MeropenemVaborbactam tested against contemporary gram-negative isolates collected worldwide during 2014, including Carbapenem-resistant, KPC-producing, multidrug-resistant, and extensively drug-resistant Enterobacteriaceae. Antimicrob Agents Chemother. 2017;61(9).

26. Hackel MA, Tsuji M, Yamano Y, Echols R, Karlowsky JA, Sahm DF. In vitro activity of the Siderophore cephalosporin, Cefiderocol, against a recent collection of clinically relevant gram-negative bacilli from North America and Europe, including Carbapenem-nonsusceptible isolates (SIDERO-WT2014 study). Antimicrob Agents Chemother. 2017:61(9).

\section{Publisher's Note}

Springer Nature remains neutral with regard to jurisdictional claims in published maps and institutional affiliations.

\section{Ready to submit your research? Choose BMC and benefit from:}

- fast, convenient online submission

- thorough peer review by experienced researchers in your field

- rapid publication on acceptance

- support for research data, including large and complex data types

- gold Open Access which fosters wider collaboration and increased citations

- maximum visibility for your research: over $100 \mathrm{M}$ website views per year

At BMC, research is always in progress.

Learn more biomedcentral.com/submissions 